\title{
Capacidade aeróbia em futebolistas de elite em função da posição específica no jogo
}

\author{
P. J. Santos \\ J. M. Soares \\ Faculdade de Ciências do Desporto e de Educação Física \\ Universidade do Porto, Porto, Portugal
}

https://doi.org/10.5628/rpcd.01.02.07

\section{RESUMO}

Vários investigadores consideram que a capacidade aeróbia desempenha um papel decisivo no futebol. Várias

investigações acerca das exigências energéticas e do padrão de actividades dos jogadores de elite durante um jogo de futebol permitiram concluir que a grande maioria das acções são marcadamente aeróbias $(4,18)$. No entanto, foram descritas diferenças acentuadas em função da posição desempenhada pelo jogador em campo (1). O propósito deste estudo foi avaliar a capacidade aeróbia dos jogadores, de acordo com a sua função, recorrendo ao limiar aeróbio-anaeróbio (12). Investigámos 91 futebolistas seniores de equipes da $1^{a}$ Liga Nacional que foram agrupados de acordo com a sua posição em jogo: centrais $(n=17)$, avançados $(n=24)$, laterais $(n=14)$ e médios $(n=36)$. A velocidade de corrida correspondente a uma concentração sanguínea de $4 \mathrm{mmol} / 1\left(\mathrm{~V}_{4}\right)$ foi considerada como medida critério na determinação do limiar aeróbio-anaeróbio. Utilizámos velocidades de corrida entre 3.0 e $4.2 \mathrm{~m} / \mathrm{s}$ com incrementos de $0.4 \mathrm{~m} / \mathrm{s}$ e patamares de carga superiores a $5 \mathrm{~min} 30$ s. Após cada patamar, o teste era interrompido durante $1 \mathrm{~min}$ para recolher sangue capilar do lóbulo da orelha e posteriormente analisado num YSI 1500L Sport. No tratamento estatístico dos dados recorremos à análise de variância a um factor e ao teste de múltipla comparação $a$ posteriori de Scheffée. O nível de significância foi estabelecido em $5 \%$. Os valores médios da $\mathrm{V}_{4}$ em cada grupo foram de $3.66 \pm 0.27 \mathrm{~m} / \mathrm{s}$ (centrais), $3.70 \pm 0.19 \mathrm{~m} / \mathrm{s}$ (avançados), $3.88 \pm 0.24 \mathrm{~m} / \mathrm{s}$ (laterais) e $3.89 \pm 0.22 \mathrm{~m} / \mathrm{s}$ (médios). Foram encontradas diferenças significativas na capacidade aeróbia entre as quatro posições $(\mathrm{F}=5.98, \mathrm{p}<0.05)$ : centrais versus médios e avançados versus médios. Em suma, os nossos dados evidenciaram diferenças significativas na capacidade aeróbia dos futebolistas em função da posição ocupada no jogo. Essas diferenças são semelhantes às descritas por outros autores relativamente à distância máxima total percorrida em jogo. Deste modo, consideramos que a determinação da $V_{4}$ pode constituir um instrumento fundamental na determinação do perfil aeróbio dos jogadores, possibilitando ainda uma individualização do treino aeróbio.

Palavras-chave: futebol, elite, capacidade aeróbia, limiar anaeróbio, lactato

\begin{abstract}
Aerobic capacity differences among soccer players according to their function in the game
\end{abstract}

Several researchers uphold the notion that aerobic capacity plays a decisive role in soccer play. In fact, investigations concerning the energy demands and the pattern of activities of elite players during a soccer match allowed to conclude that most of the actions are markedly aerobic $(4,18)$. Nevertheless, considerable differences have been reported according to the player's position in the game (1). Thus the purpose of this study was to evaluate the player's aerobic capacity according to their function using the aerobic-anaerobic threshold (12). We investigated 91 Portuguese top male senior soccer players from several 1 st League teams which were grouped according to their function in the game: defenders $(n=17)$, forwards $(n=24)$, full-backs $(n=14)$ and mid-fielders $(n=36)$. The running speed at a blood lactate concentration of $4 \mathrm{mmol} / 1\left(\mathrm{~V}_{4}\right)$ was used as criterion for the aerobic-anaerobic threshold determination. We've used running speeds ranging from 3.0 to $4.2 \mathrm{~m} / \mathrm{s}$ with increments of $0.4 \mathrm{~m} / \mathrm{s}$ and steps above $5 \mathrm{~min} 30 \mathrm{~s}$. Following each loading level the test was interrupted for $1 \mathrm{~min}$ to take blood samples from the ear lobe which were analysed using an YSI 1500L Sport. Statistical analysis included Anova and a Scheffe post hoc test. Mean results in the $\mathrm{V}_{4}$ for each group was $3.66 \pm 0.27 \mathrm{~m} / \mathrm{s}$ (defenders), $3.70 \pm 0.19 \mathrm{~m} / \mathrm{s}$ (forwards), $3.88 \pm 0.24 \mathrm{~m} / \mathrm{s}$ (full-backs) and $3.89 \pm 0.22 \mathrm{~m} / \mathrm{s}$ (mid-fielders). Significant results were achieved concerning the $\mathrm{V}_{4}$ among the four game positions $(\mathrm{F}=5.98, \mathrm{p}<0.05)$ : defenders versus mid-fielders and forwards versus mid-fielders. In conclusion, our data evidenced significant differences in aerobic capacity according to the player's function in the game, which are similar to those reported by other authors in relation to the distance covered during a match. We consider that these results strongly suggest that $\mathrm{V}_{4}$ determination might be seen both as an important tool for determining soccer player's aerobic profile and for allowing the individualisation of aerobic training.

Key words: soccer, elite, aerobic capacity, anaerobic threshold, lactate 


\section{INTRODUÇÃO}

O futebol é um jogo extremamente complexo do ponto de vista fisiológico, com acções específicas que evidenciam uma tipologia de esforço de grande diversidade e que, em termos metabólicos, apelam a fontes energéticas claramente distintas. De facto, o futebolista, dada a natureza intermitente do seu esforço e a ampla faixa de intensidades que o caracteriza, tem de privilegiar no seu treino aspectos tão distintos como o desenvolvimento da força explosiva, da velocidade, da resistência anaeróbia e da resistência aeróbia.

Entre os distintos sistemas energéticos que sustentam as acções de jogo, vários têm sido os autores que referem o metabolismo aeróbio como constituindo o suporte energético fundamental para uma partida de futebol. Com efeito, investigações acerca das exigências energéticas e do padrão de actividades evidenciados pelos jogadores de elite durante um jogo, permitiram concluir que a maioria das acções são acentuadamente aeróbias e suportadas pelo metabolismo oxidativo $(4,18)$. A distância média total percorrida pelos jogadores durante uma partida de $90 \mathrm{~min}$ situa-se em torno dos $11 \mathrm{~km}(4,23)$, o que vem reforçar a noção de que, para um futebolista de topo, é fundamental possuir uma boa preparação aeróbia.

A este propósito, têm sido descritas diferenças acentuadas da performance aeróbia nestes jogadores consoante a sua função em campo $(1,5,7)$. No entanto, a quase totalidade destas investigações tem sido conduzida em laboratório, razão pela qual os resultados obtidos pouca aplicabilidade têm no trabalho de campo do atleta.

Com base no anteriormente exposto, foi objectivo principal deste estudo investigar eventuais diferenças a nível da capacidade aeróbia entre futebolistas de elite, de acordo com a sua função no jogo (defesas, centrais, médios e laterais). Para a avaliação aeróbia dos sujeitos recorremos ao limiar aeróbio-anaeróbio, mas utilizámos um teste de terreno de simples execução e, simultaneamente, capaz de fornecer dados transferíveis para o treino dos atletas.

\section{MATERIAL E MÉTODOS}

A amostra deste estudo foi constituída por 91 futebolistas seniores portugueses pertencentes a equipas da $1^{\text {a }}$ Liga.

Recorremos ao limiar aeróbio-anaeróbio para determinar a capacidade aeróbia dos jogadores, tendo utilizado um teste de terreno realizado numa pista sintética de atletismo $(400 \mathrm{~m})$ com 4 patamares de intensidade crescente. As distâncias situaram-se entre $1200 \mathrm{~m}$ e $1600 \mathrm{~m}$ (fig. I), tendo os atletas corrido sempre a uma velocidade constante durante um período nunca inferior a 5'30".

Para assegurar um ritmo de corrida uniforme foram assinalados os tempos de passagem (avisos sonoros) em cada $200 \mathrm{~m}$ durante a realização dos patamares de carga. As velocidades utilizadas variaram entre $3.0 \mathrm{e}$ $4.6 \mathrm{~m} / \mathrm{s}$ e os incrementos de carga foram de $0.4 \mathrm{~m} / \mathrm{s}$ por patamar (quadro I).

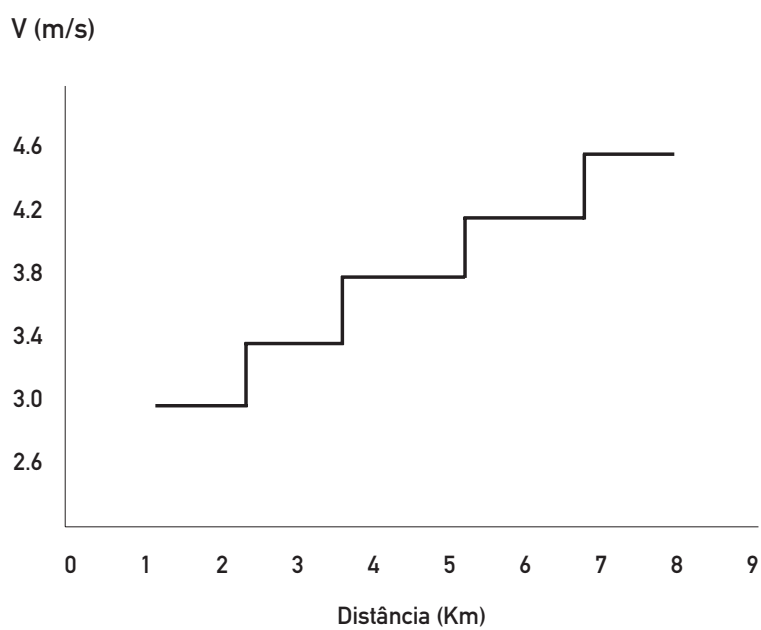

Fig. I - Representação gráfica da dinâmica de carga referente ao teste de terreno utilizado na avaliação da capacidade aeróbia dos futebolistas e andebolistas. Foram utilizadas distâncias de $1200 \mathrm{~m}$ $(3.0$ e $3.4 \mathrm{~m} / \mathrm{s})$ e $1600 \mathrm{~m}(3.8,4.2$ e $4.6 \mathrm{~m} / \mathrm{s})$ por patamar e incrementos de $0.4 \mathrm{~m} / \mathrm{s}$. 


\begin{tabular}{|c|c|c|c|c|}
\hline$v(\mathrm{~m} / \mathrm{s})$ & $400 \mathrm{~m}$ & $800 \mathrm{~m}$ & $1200 \mathrm{~m}$ & $1600 \mathrm{~m}$ \\
\hline 3.0 & 2'13"33 & 4’26"67 & 6'40"00 & \\
\hline 3.4 & 1’57"65 & 3’55"29 & 5’52"94 & \\
\hline 3.8 & 1’45"26 & 3’30"53 & 5’15"79 & 7’01"05 \\
\hline 4.2 & 1’35"24 & 3'10"48 & $4^{\prime} 45^{\prime \prime} 71$ & 6’20"95 \\
\hline 4.6 & 1’26"96 & 2’53"91 & 4'20"87 & $5^{\prime} 47 " 83$ \\
\hline
\end{tabular}

Quadro I - Tabela utilizada nos testes de terreno com indicação dos respectivos tempos de passagem (cada $400 \mathrm{~m}$ ) para as várias velocidades $(\mathrm{m} / \mathrm{s})$ correspondentes aos patamares de carga utilizados.

No final de cada percurso o teste era interrompido durante 1 min para recolher sangue capilar do lóbulo da orelha para determinação imediata da concentração sanguínea de lactato utilizando um YSI-1500L Sport. O teste era dado como concluído quando o atleta ultrapassava uma concentração sanguínea de $4 \mathrm{mmol} / 1$ de lactato.

Como critério de detecção do limiar aeróbioanaeróbio utilizámos a velocidade de corrida correspondente a uma concentração sanguínea de $4 \mathrm{mmol} / \mathrm{l}\left(\mathrm{V}_{4}\right)$ determinada por interpolação linear a partir dos dados obtidos nos testes de terreno. No tratamento estatístico dos dados, além da determinação dos valores médios e do desvio padrão, foram utilizados a análise de variância a um factor e o teste de múltipla comparação a posteriori de Scheffée. O nível de significância foi estabelecido em $5 \%$.

\section{RESULTADOS}

Os valores médios para a amostra relativos à idade, altura e peso foram de $25 \pm 2.6$ anos, $177.8 \pm 4.1 \mathrm{~cm} \mathrm{e}$ $72.8 \pm 4.5 \mathrm{~kg}$, respectivamente. O valor médio correspondente ao limiar aeróbio-anaeróbio foi de $3.85 \pm 0.28 \mathrm{~m} / \mathrm{s}$. Os resultados da ANOVA para a posição ocupada pelos jogadores evidenciou diferenças estatisticamente significativas $(\mathrm{F}=5.98, \mathrm{p}<0.05)$. Os valores médios da $\mathrm{V} 4$ por posição no jogo podem ser observados na fig. II. O teste de múltipla comparação a posteriori expressou diferenças significativas somente entre médios vs centrais e médios vs avançados.

$\mathrm{V} 4(\mathrm{~m} / \mathrm{s})$

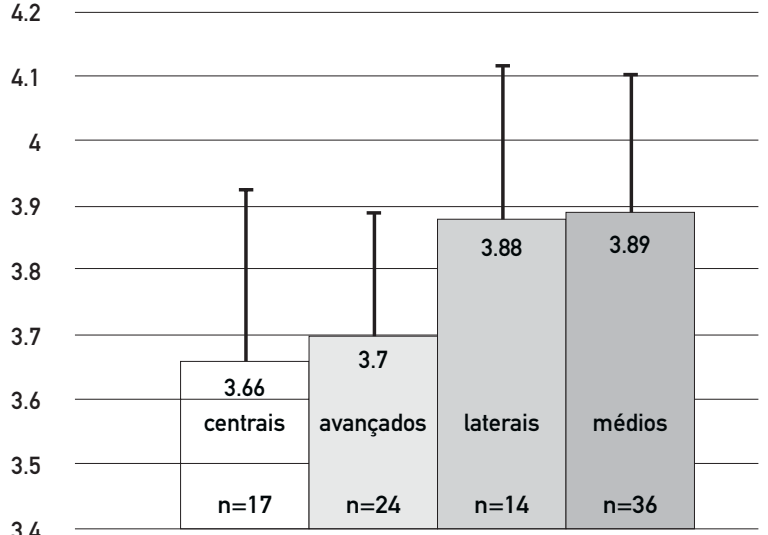

Fig.II - Comparação entre os valores médios $( \pm d p)$ da $V_{4}$ (expressa em $\mathrm{m} / \mathrm{s}$ ) no grupo de futebolistas em função da posição ocupada pelos jogadores na equipa.

\section{DISCUSSÃO}

Vários estudos realizados nos últimos 10 anos $\mathrm{e}$ centrados na análise de jogo têm evidenciado que um jogador de futebol percorre em jogo uma distância total de 10 a $12 \mathrm{Km}(3,4,6,13,15,17,22)$. No entanto, sabe-se que os vários elementos de uma equipa percorrem distâncias bem diferentes de acordo com a sua função no jogo $(4,22)$. Ao longo das últimas décadas verificou-se um aumento progressivo da distância total percorrida (DTP) em jogo, tendo sido referido, a partir da análise das distâncias percorridas em campeonatos do mundo, que esse valor aumentou mais de $100 \%$ entre os mundiais da Suiça (1954) e de Itália (1990), ou seja, mais especificamente, de $4500 \mathrm{~m}$ para $10000 \mathrm{~m}$, respectivamente (21). Vários autores têm igualmente descrito níveis diferenciados de condição aeróbia em futebolistas consoante a sua posição no jogo. Com efeito, estudos acerca da potência máxima aeróbia em futebolistas revelaram níveis diferentes de acordo com a função desempenhada $(1,5,7,16)$. Uma investigação recente (20) refere mesmo que a DTP em jogo depende de forma significativa da capacidade aeróbia dos jogadores e que $60 \%$ da DTP pode ser explicada pelo nível aeróbio do atleta. Em suma, os dados da literatura sugerem que jogadores 
de diferentes posições não só percorrem distâncias diferentes, como igualmente apresentam performances aeróbias distintas.

No entanto, são escassos os estudos nesta modalidade em que tenham sido utilizados testes de terreno específicos para avaliar a performance aeróbia dos sujeitos. Uma investigação recente (14) utilizando o Yo-Yo intermittent endurance test (2), revelou diferenças significativas de resistência entre laterais e médios versus centrais e avançados $(\mathrm{n}=327)$. Porém, não conhecemos nenhuma investigação no terreno que tenha procurado determinar as diferenças a nível da capacidade aeróbia por posição com base no limiar anaeróbio. Com efeito, apesar de Bangsbo (1) ter utilizado o limiar anaeróbio num estudo com objectivos semelhantes, fê-lo, contudo, recorrendo à investigação laboratorial. A grande vantagem da investigação no terreno é, obviamente, o grande transfere que permite dessa informação para o trabalho de campo do atleta. Deste modo, possibilita não apenas a recolha de dados fundamentais para a caracterização fisiológica dos jogadores, como permite ainda a obtenção de dados preciosos para a equipa técnica, no sentido de rentabilizar, tanto quanto possível, o treino desses atletas. Adicionalmente, quanto mais investigação for efectuada com base neste tipo de testes, tanto maior será a hipótese de fazer a validação cruzada dos dados de forma a optimizar a metodologia utilizada neste tipo de pesquisas.

Relativamente ao valor médio da $\mathrm{V}_{4}$ obtida pelos futebolistas da nossa amostra $(3.85 \mathrm{~m} / \mathrm{s})$, verificámos que foi muito semelhante à descrita num estudo similar (8) envolvendo tanto jogadores amadores $(n=15)$ como profissionais $(n=15)$ do campeonato alemão. Nessa investigação não foram encontradas diferenças significativas da capacidade aeróbia entre grupos, com os profissionais a apresentarem um valor de $3.71 \mathrm{~m} / \mathrm{s}$ e os amadores $3.90 \mathrm{~m} / \mathrm{s}$. Outras investigações realizadas com selecções nacionais alemãs referem valores médios de $4.15 \mathrm{~m} / \mathrm{s}$ e de $4.05 \mathrm{~m} / \mathrm{s}(10,11)$. No entanto, esses testes foram realizados em laboratório e utilizando patamares de $3 \mathrm{~min}$, o que implica uma sobrevalorização da $V_{4}$ (9) relativamente à utilização de patamares de $5 \mathrm{~min}$ (ou superiores).
Em relação aos resultados obtidos em função da posição ocupada na equipa, os valores mais elevados encontrados nos médios $(3.89 \mathrm{~m} / \mathrm{s})$ e laterais $(3.88 \mathrm{~m} / \mathrm{s})$ versus avançados $(3.70 \mathrm{~m} / \mathrm{s})$ e centrais $(3.66 \mathrm{~m} / \mathrm{s})$, são algo semelhantes aos descritos por Bangsbo (1) que também refere valores de resistência superiores nos médios relativamente aos centrais, embora utilizando como referência a velocidade de corrida (tapete rolante) correspondente a uma concentração sanguínea de lactato de $3 \mathrm{mmol} / 1\left(\mathrm{~V}_{3}\right)$.

A melhor $\mathrm{V}_{4}$ evidenciada pelos médios resulta, provavelmente, da função de ligação entre defesas e atacantes, o que implica um maior volume de corrida sustentada $(1,4)$. Com efeito, pensamos que as diferenças significativas encontradas entre médios versus centrais e médios versus avançados, poderão, entre outros factores, estar relacionadas com a maior distância percorrida tanto em treino como em competição, em resultado da especificidade das funções desempenhadas.

Também os resultados da literatura a nível da potência aeróbia em futebolistas de alto nível $(1,16)$ referem valores mais elevados nos médios, comparativamente a defesas centrais e guarda-redes. Resultados concordantes são descritos num outro estudo (19) com uma amostra que incluía jogadores portugueses da $1^{\text {a }}$ divisão nacional $(n=44)$, com os valores mais elevados a serem exibidos pelos médios e laterais (59.5 e $59.3 \mathrm{ml} / \mathrm{min} / \mathrm{Kg}$, respectivamente) relativamente aos centrais $(56.8 \mathrm{ml} / \mathrm{min} / \mathrm{Kg})$ e avançados $(54.9 \mathrm{ml} / \mathrm{min} / \mathrm{Kg})$.

Os dados emergentes desta investigação permitiram constatar que, em termos fisiológicos, as exigências impostas a jogadores que ocupem posições diferentes no terreno de jogo podem ser, do ponto de vista aeróbio, substancialmente distintas. Futuras pesquisas realizadas no terreno com base no limiar anaeróbio permitirão, entre outras coisas, a elaboração de tabelas com valores de referência por posição. Dada a grande aplicabilidade prática deste tipo de trabalhos, consideramos que eles podem constituir uma mais valia tanto em termos de investigação, como nos aspectos relacionados com o controlo de treino dos atletas.

Adicionalmente, dados provenientes de outras investigações parecem ainda sugerir que os 
futebolistas que percorrem maiores distâncias em situação de jogo são, simultaneamente, aqueles que exibem níveis superiores de capacidade aeróbia (20). Deste modo, se aceitarmos que a DTP pode ser um factor importante no jogo, então seguramente que o desenvolvimento da capacidade aeróbia será um factor a contemplar no planeamento de treino de uma equipa de futebol. Nesta perspectiva, o conhecimento da $\mathrm{V}_{4}$ por posição pode ser um instrumento importante para a equipa técnica no sentido de ultrapassar eventuais carências a nível das capacidades físicas de alguns jogadores.

Em suma, os nossos dados evidenciaram diferenças significativas na capacidade aeróbia dos futebolistas em função da posição ocupada no jogo. Essas diferenças são semelhantes às descritas por outros autores tanto em relação à DTP em jogo, como aos valores de potência e de capacidade aeróbias. Adicionalmente, consideramos que a determinação da $V_{4}$ através de um simples teste de terreno pode constituir um instrumento fundamental na determinação do perfil aeróbio dos jogadores, possibilitando ainda uma individualização do treino aeróbio por permitir uma aplicação quase imediata dos dados obtidos no trabalho de campo dos atletas.

\section{CORRESPONDÊNCIA}

\section{Paulo Jorge Miranda Santos}

Faculdade de Ciências do Desporto

e de Educação Física

Universidade do Porto

Rua Dr. Plácido Costa, 91

4200.450 Porto

Portugal

[psantos@fcdef.up.pt] 


\section{REFERÊNCIAS}

1. Bangsbo J. (1993). The physiology of soccer Dissertação apresentada às provas de doutoramento. Universidade de Copenhaga. Copenhaga

2. Bangsbo J. (1996). Yo-Yo tests. HO+Storm, Copenhagen, Denmark and Tocano Music A/S, Smorum, Denmark

3. Bangsbo J, Lindquist F (1992). Comparison of various exercise tests with endurance performance during soccer in professional players. Int J Sports Med 13:152-157

4. Bangsbo J, Norregaard L, Thorsoe F (1991) Activity profile of competition soccer. Can J Sports Sci 16:110-116

5. Davis J, Brewer J, Atkin D (1992). Pre-season physiological characteristics of English first and second division soccer players. J Sports Sci 10(6):541-547

6. D’Ottavio S, Tranquili C (1992). La prestazione del giocatore di calcio. SDS-Rivista di Cultura Sportiva 24:74-78 7. Faina M, Gallozzi C, Lupo S, Colli R, Sassi R, Marini C (1988). Definition of the physiological profile of the soccer player. In: Reilly T, Lees A, Davis K, Murphy (Ed.) Science and Football - Proceedings $1^{\text {st }}$ World Congress of Science and Football. London. 158-163

8. Föhrenbach R, Buschmann J, Mader A, Hollmann W (1993). Speed and endurance in soccer: a comparison of professional and amateur players. Sci Foot, 7:24-30

9. Heck H, Mader A, Hess G, Mücke S, Müller R, Hollmann W (1985). Justification of the $4 \mathrm{mmol} / \mathrm{l}$ lactate threshold. Int $J$ Sports Med 6:117-130

10. Hollmann W, Liesen H, Mader A, Heck H, Rost R, Dufaux B, Schürch P, Lagerström D, Föhrenbach R (1981). Zur Höchst- und Dauerleistungsfähigkeit der deutschen FußballSpitzenspieler. Deutsch Zschr Sportmed 52:113-120

11. Liesen H, Ludemann E, Schmengler D, Föhrenbach R, Mader A (1985). Trainingssteuerung im Hochleistungssport: einige Aspekte und Beispiele. Dtsch Ztschr Sportmed 36:8-18 12. Mader A, Liesen H, Heck H, Philippi H, Rost R, Schürch P, Hollmann W (1976). Zur Beurteilung der sportartspezifischen Ausdauerleistungsfähigkeit im Labor. Sportarzt. Sportmed. 24(4), 80(5), 26(5):109
13. Maréchal R (1996). Exigences métaboliques et cardiaques du match de football. Sport 154:36-39

14. Oliveira JM (2000). Avaliação da resistência em desportos de esforço intermitente. Dissertação apresentada às provas de doutoramento. Faculdade de Ciências do Desporto e de Educação Física da Universidade do Porto. Porto 15. Pirnay F, Geurd P, Maréchal R (1991). Contraintes physiologiques d'un match de football. Sport 2:71-79 16. Puga N, Ramos J, Agostinho J, Lomba I, Costa O (1991). Physiological profile of a $1^{\text {st }}$ division Portuguese professional football team. In: Reilly T, Clarys J, Stibee A. (Ed.) Science and Football lll. London. 40-42

17. Rebelo A (1993). Caracterização da actividade física do futebolista em competição. Dissertação apresentada às provas de Aptidão Pedagógica e de Capacidade Científica. Faculdade de Ciências do Desporto e de Educação Física da Universidade do Porto. Porto

18. Reilly T (1990). Football. In: Reilly T., Secher N., Snell P. and Williams C. (Ed.) Physiology of sports. 372-401, London 19. Santos JA (1995). Estudo comparativo, fisiológico, antropométrico e motor entre futebolistas de diferente nível competitivo e velocistas, meio-fundistas e fundistas de atletismo. Dissertação apresentada às provas de doutoramento. Faculdade de Ciências do Desporto e de Educação Física da Universidade do Porto. Porto

20. Santos P, Valente A, Soares J (2001). Aerobic capacity versus total distance covered during a game in elite soccer players. Med Sci Sports Exerc 33(5):S157

21. Serrano M (1993). Consideraciones a las tendencias del fútbol actual. El Entrenador Español (fútbol) 57:24-28

22. Valente A (2000). Limiar aeróbio-anaeróbio e distância percorrida em jogo. Dissertação apresentada às provas de Mestrado em Desporto de Alto Rendimento. Faculdade de Ciências do Desporto e de Educação Física da Universidade do Porto. Porto

23. Withers RT, Maricic Z, Wasilewski S, Kelly L (1982). Match analysis of Australian professional soccer players. J Hum Mov Stud 8:159-176 